\title{
Synthesis of Some Novel and Potent Anti-Plasmodial Aminoalkyl Chalcone Derivatives
}

\author{
Pravin Kendrekar 1,* (D), Samson Mashele ${ }^{1}$, Sunil Tekale 2(D), Rajandra Pawar 2,*(D) \\ 1 Unit for Drug Discovery Research (UDDR), Department of Health Sciences Faculty of Health and Environmental \\ Sciences, Central University of Technology, Free State (CUT) Private Bag X20539, Bloemfontein, 9300, South Africa \\ 2 Department of Chemistry, Deogiri College, Aurangabad-431005, MS, India \\ * Correspondence: kkpravin@gmail.com (P.K.); rppawar@yahoo.com (R.P.);
}

Scopus Author ID 56095571700

Received: 22.03.2020; Revised: 28.04.2020; Accepted: 29.04.2020; Published: 02.05.2020

\begin{abstract}
Malaria remains to be a health and an economic burden to many people living in Sub-Sahara and Africa. According to World health Organization (WHO) in 2017, 219 million cases of malaria worldwide were documented. Its increase by 2 million from the year 2016 resulted in 435 thousand deaths every day among 1190 deaths of young children more than cases 5 per day have been reported. Among the different species of plasmodium parasite, Plasmodium falciparum is mainly responsible for causing malaria in Africa whereas Plasmodium vivax is the most prevalent in countries outside Africa. Africa suffers from malaria a lot. Almost half of the world's population is at risk of contracting malaria and approximately $90 \%$ of the death cases of Malaria in the world appear from Africa. In our research group in past, we have established that chalcones with an aminoalkyl moiety on one of the aromatic rings have promising in vitro antimalarial activity. We successively enhanced the bioavailability from $3 \%$ to $25 \%$ in mice model with the derivatization of the potential leads with the help of substitutions of the function groups at 4- position from $-\mathrm{F}$ to $-\mathrm{CF}_{3}$.
\end{abstract}

Keywords: Synthesis; Characterizations; Chalcones; Bioavailability.

(C) 2020 by the authors. This article is an open access article distributed under the terms and conditions of the Creative Commons Attribution (CC BY) license (https://creativecommons.org/licenses/by/4.0/).

\section{Introduction}

Malaria remains to be a health and an economic burden to many people living in the world; particularly in Asia, Sub-Sahara and Africa [1-5]. Due to drug resistance against many current treatments, including the first-line treatment based on the plant derived compound, artemisinin, there is a dire need to identify new antimalarial medicines [6-10]. We have established that chalcones with an aminoalkyl moiety (Scheme 1) on one of the aromatic rings have promising in vitro antimalarial activity.

The Mannich reaction was used to introduce the aminoalkyl moiety. This reaction requires an aromatic $\mathrm{OH}$ at the ortho position. We have established that chalcones with an aminoalkyl moiety on one of the aromatic rings have promising in vitro antimalarial. This finding supports our hypothesis that nitrogen containing flavonoids will have enhanced biological activity as compared to naturally occurring non nitrogen containing flavonoids. We synthesize a total of 60 compounds in an effort to enhance bioactivity, reduce toxicity and increase oral bioavailability. Some of our compounds have similar or lower IC 50 values than chloroquine against chloroquine sensitive malaria strains (D10, NF54).

There is little difference in activity against chloroquine sensitive and chloroquine resistant strains (Dd2, K1) as is evident from the small differences in IC50 values. 
This activity against drug resistant malaria strains is probably to be expected as our compounds are novel with structures totally unrelated to the currently used antimalarial drug. Our reported antimalarial compounds are relatively non-complicated and inexpensive to manufacture and promise cheap antimalarial drugs [11-12]. Toxicity test (in vitro CHO cell assays) suggests that our compounds are relatively nontoxic with high SI indices. This is supported by initial in vivo tests on mice which heaved no adverse effects after dosage with selected analogs. Initial in vivo bioavailability determinations afforded poor results (3\%). Efforts to enhance bioavailability via protecting the phenolic $\mathrm{OH}$ group against first pass metabolism (prodrug strategy) failed because the $\mathrm{OH}$ group resisted ether or ester formation. We attribute this to hydrogen bonding of the ortho $\mathrm{OH}$ to the ortho aminoalkyl group. Further investigations indicated that bulky groups on the A-ring increase bioavailability. Our best candidate with large moieties on the Aring so far has a bioavailability of $25 \%$. Further work is in progress to increase the bioavailability of our compounds to a standard that is attractive to pharmaceutical companies in the near future.

\section{Materials and Methods}

All chemicals were purchased from Sigma-Aldrich and used as received. Melting points were recorded in open capillaries. ${ }^{1} \mathrm{H}$ NMR spectra were recorded in $\mathrm{CDCl}_{3}$ on a Bruker BioSpin spectrometer at $600 \mathrm{MHz}$ using TMS as an internal standard. Mass spectra ESIMS were recorded and IR spectra were recorded on a Shimadzu FTIR spectrometer in $\mathrm{KBr}$ pallets.

\subsection{General Procedure for the synthesis of Aminoalkylated Chalcones via the Mannich} reaction.

A mixture of the appropriate chalcone (1 eq.), paraformaldehyde (1.5 eq.), and the appropriate amine (2 eq.) was dissolved in $\mathrm{EtOH}(2 \mathrm{~mL})$ and conc. $\mathrm{HCl}$ ( 5 drops). The reaction mixture was refluxed for 9 hours until TLC (Toluene: Acetone 7:3) showed the disappearance of the starting material. The reaction mixture was quenched with solid $\mathrm{NaHCO}_{3}$ and extracted with EtOAc $(2 \times 50 \mathrm{~mL})$ and water $(2 \times 50 \mathrm{~mL})$. The organic layers were combined, dried by using anhydrous $\mathrm{Na}_{2} \mathrm{SO}_{4}$ and concentrated under reduced pressure to afford crude product. The crude product was further purified by column chromatography. During this study, several substituents on ring $\mathrm{A}$ such as $-\mathrm{H},-\mathrm{CH}_{3},-\mathrm{C}_{2} \mathrm{H}_{5},-\mathrm{F}$ and $-\mathrm{CF}_{3}$ were employed.

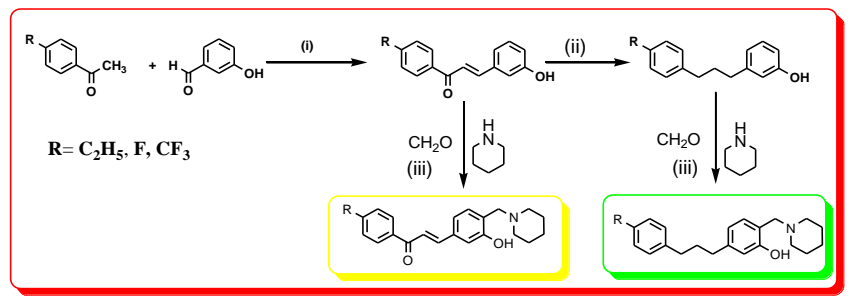

Scheme 1. Reagents and conditions: (i) $50 \% \mathrm{KOH}$-solution, EtOH, rt; (ii) $\mathrm{Pd}(\mathrm{OH})_{2} / \mathrm{C}, \mathrm{H}_{2}, \mathrm{EtOAc}: \mathrm{H}_{2} \mathrm{O}$, (iii) $\mathrm{EtOH} \& \mathrm{HCl}$ reflux $(9 \mathrm{~h})$.

\subsection{Bioassay.}

Antimalarial activity screening of the synthesized compounds was used to determine the potential of these compounds as sources of antimalarial compounds [13]. A good source of antimalarial compounds should inhibit parasites selectively and be harmless towards other cells. Cytotoxicity experiments were used to determine general toxic properties of compounds against living cells. Antimalarial activity and cytotoxicity information are used to determine 
selectivity indexes, which are used as a guide to determine the potential of a compound for further investigation. An active antimalarial compound should be at least tenfold more active against the targeted organism than against mammalian cells to be considered for further testing. Cytotoxicity of the synthesized compounds was tested against Chinese hamster ovarian (CHO) cells which were cultured according to a standard operating procedure prepared by the collaborators. The MTT assay as described by Mosmann (with minor modifications) was used to determine cell viability [14].

\subsection{Experimental procedure.}

2.3.1. Synthesis of 5-(3-(4-fluorophenyl)propyl)-2-(piperidin-1-ylmethyl)phenol (A).

Compound (A) was synthesized according to the general procedure using 3-(3-(4fluorophenyl)propyl)phenol $(0.200 \mathrm{~g} ; 0.87 \mathrm{mmol})$, paraformaldehyde $(0.052 \mathrm{~g} ; 1.73 \mathrm{mmol})$, and piperidine $(0.18 \mathrm{~mL} ; 1.84 \mathrm{mmol})$ as starting materials. The crude reaction mixture was chromatographed (TLC, Toluene: Acetone 7:3). The synthesized compounds were well characterized in our previous studies [11, 15]. The fraction $\mathrm{R}_{\mathrm{f}} 0.50$ yielded 5-(3-(4fluorophenyl)propyl)-2-(piperidin-1-ylmethyl)phenol (A) as a yellow oil (0.269 g, 95\%) (Figure 1).

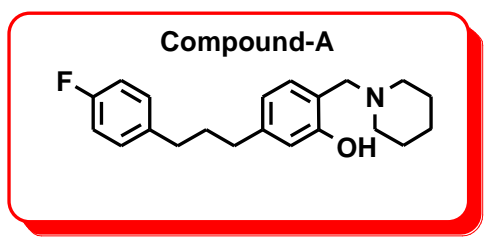

Figure 1. Structure of 5-(3-(4-fluorophenyl)propyl)-2-(piperidin-1-ylmethyl)phenol.

${ }^{1} \mathrm{H}$ NMR (600 MHz, $\left.\mathrm{CDCl}_{3}, \mathrm{Me}_{4} \mathrm{Si}\right) \delta$ ppm: 1.64 - 1.32 (6H, H-3'", H-4'", H-5"'), $1.87-$ 1.78 (2H, m, H-2), 2.41 - 2.04 (4H, H-2'", H-6'"), 2.50 - 2.46 (2H, m, H-2), 2.55 - 2.51, (2H, m, H-3), 3.55 (2H, s, CH $), 6.50$ (1H, dd, J = 7.6, $1.3 \mathrm{~Hz}, \mathrm{H}-6 "), 6.57$ (1H, d, J = 1.3 Hz, H-2"), $6.78(1 \mathrm{H}, \mathrm{d}, \mathrm{J}=7.6 \mathrm{~Hz}, \mathrm{H}-5 "), 6.87(2 \mathrm{H}, \mathrm{t}, 3 \mathrm{JH}-\mathrm{H}=8.6 \mathrm{~Hz}$; 4JH-F = 8.6 Hz, H-3', H-5'), 7.05 $\left(2 \mathrm{H}, \mathrm{dd}, 3 \mathrm{JH}-\mathrm{H}=8.6 \mathrm{~Hz} ; 4 \mathrm{JH}-\mathrm{F}=5.5 \mathrm{~Hz}, \mathrm{H}-2\right.$ ', H-6'); ${ }^{13} \mathrm{C}$ APT NMR $\left(150 \mathrm{MHz}, \mathrm{CDCl}_{3}\right.$, $\mathrm{Me}_{4} \mathrm{Si}$ ) $\delta$ ppm 24.0 (C-4"'), 25.9 (C-3"', C-5'"), 32.9 (C-2), 34.6 (C-1), 35.1 (C-3), 53.9 (C-2'", C-6"'), 61.9 (CH2), 115.0 (2C, d, ${ }^{2} \mathrm{~J}_{\mathrm{C}-\mathrm{F}}=20.9 \mathrm{~Hz}, \mathrm{C}-3$ ', C-5'), 116.0 (C-2"), 119.0 (C-6"), 119.1 (C-4"), 128.3 (C-2"), 129.7 (2C, d, ${ }^{3} \mathrm{~J}_{\mathrm{C}-\mathrm{F}}=7.7 \mathrm{~Hz}, \mathrm{C}-2$ ', C-6'), $138.0\left(1 \mathrm{C}, \mathrm{d},{ }^{4} \mathrm{~J}_{\mathrm{C}-\mathrm{F}}=3.2 \mathrm{~Hz}, \mathrm{C}-\right.$ $\left.1^{\prime}\right), 142.8$ (C-1"), 158.0 (C-3"), 161.2 (1C, d, $\left.{ }^{1} \mathrm{~J}_{\mathrm{C}-\mathrm{F}}=243.1 \mathrm{~Hz}, \mathrm{C}-4{ }^{\prime}\right) ;{ }^{19} \mathrm{~F}$ NMR $\delta(282.4 \mathrm{MHz}$, $\mathrm{CDCl}_{3}, \mathrm{C}_{6} \mathrm{~F}_{6}$ ) -118.1 (s, F); IR (neat): $v_{\max } \mathrm{cm}^{-1}=819.83,1218.86,1508.34,2934.84$; Found $\left(\right.$ TOF MS ES) $[\mathrm{M}+\mathrm{H}]^{+} 328.2073,\left(\mathrm{C}_{21} \mathrm{H}_{26} \mathrm{FNO}+\mathrm{H}^{+}\right): m / z 328.2077$; HPLC purity $96.9 \%$, $\mathrm{t}_{\mathrm{R}}$ $=1.62 \mathrm{~min}$.

\subsubsection{Synthesis of 5-(3-(4-ethylphenyl)propyl)-2-(piperidin-1-ylmethyl)phenol (B).}

Compound (B) was synthesized using 3-(3-(4-ethylphenyl)propyl)phenol (0.100 g; $0.42 \mathrm{mmol})$, paraformaldehyde $(0.076 \mathrm{~g} ; 2.53 \mathrm{mmol})$ and piperidine $(0.10 \mathrm{~mL} ; 1.00 \mathrm{mmol})$ according to the general procedure. The crude reaction mixture was chromatographed (TLC, Toluene: Acetone 7:3). The fraction $\mathrm{R}_{\mathrm{f}} 0.50$ yielded 5-(3-(4-ethylphenyl)propyl)-2-(piperidin1-ylmethyl)phenol (B) as a yellow oil (0.087 g, 62\%) (Figure 2). ${ }^{1} \mathrm{H} \mathrm{NMR}\left(600 \mathrm{MHz}, \mathrm{CDCl}_{3}\right.$, $\left.\mathrm{Me}_{4} \mathrm{Si}\right) \delta \mathrm{ppm} 1.14\left(3 \mathrm{H}, \mathrm{t}, \mathrm{J}=7.6 \mathrm{~Hz}, \mathrm{CH}_{3}\right), 1.38(2 \mathrm{H}$, broadend s, 1 x N-CH$), 1.58-1.51$ $\left(4 \mathrm{H}, \mathrm{m}, 2 \mathrm{x} \mathrm{N}-\mathrm{CH}_{2}\right), 1.89-1.80(2 \mathrm{H}, \mathrm{m}, \mathrm{H}-2), 2.26-2.02\left(2 \mathrm{H}, \mathrm{m}, \mathrm{CH}_{3}-\mathrm{CH}_{2}\right), 2.57-2.29(8 \mathrm{H}$, 
m, H-1, H-3, 2 x N-CH2), 3.54 (2H, s, CH2), 6.51 (1H, dd, J = 7.6, 1.4 Hz, H-6"), 6.58 (1H, d, $\mathrm{J}=1.4 \mathrm{~Hz}, \mathrm{H}-2 "), 6.77$ (1H, d, J = 7.6 Hz, H-5"), 7.03 (4H, s, H-2', H-6', H-3', H-5'); ${ }^{13} \mathrm{C}$ NMR $\left(150 \mathrm{MHz}, \mathrm{CDCl}_{3}, \mathrm{Me}_{4} \mathrm{Si}\right) \delta \mathrm{ppm}: 15.7\left(\mathrm{CH}_{3}\right), 24.1$ (C-4'"), 25.9 (C-3"', C-5"'), 28.5 ( $\mathrm{CH}_{3}-$ $\mathrm{CH}_{2}$ ), 32.8 (C-2), 35.0 (C-1), 35.2 (C-3), 53.9 (C-2"', C-6"'), $62.0\left(\mathrm{CH}_{2}\right), 116.0$ (C-2"), 119.0 (C-4"), 119.1 (C-6"), 127.8 (C-3', C-5'), 128.3 (C-5"), 128.4 (C-2', C-6'), 139.6 (C-1'), 141.5 (C-1"), 143.1 (C-4'), 158.0 (C-3"); IR (neat): $v_{\max } \mathrm{cm}^{-1}=782.72,1390.20,1452.40,2919.13$; Found (TOF MS ES) $[\mathrm{M}+\mathrm{H}]^{+} 338.2486,\left(\mathrm{C}_{23} \mathrm{H}_{31} \mathrm{NO}+\mathrm{H}^{+}\right): \mathrm{m} / z$ 338.2484. HPLC purity $99.4 \%$, $\mathrm{t}_{\mathrm{R}}=1.75 \mathrm{~min}$.

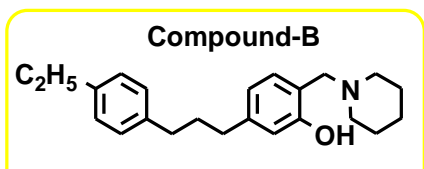

Figure 2. Structure of 5-(3-(4-ethylphenyl)propyl)-2-(piperidin-1-ylmethyl)phenol.

2.3.3. Synthesis of 2-(piperidin-1-ylmethyl)-4-(3-(4-(trifluoromethyl)phenyl)propyl)phenol (C).

Compound (C) was synthesized according to the general procedure using 4-(3-(4(trifluoromethyl)phenyl)propyl)phenol (116)(0.050 g; $0.18 \mathrm{mmol})$, paraformaldehyde $(0.052$ $\mathrm{g} ; 1.73 \mathrm{mmol})$, and piperidine $(0.18 \mathrm{~mL} ; 1.84 \mathrm{mmol})$ as starting materials. The crude reaction mixture was separated by flash column chromatography (H: EtOAc 6:4, $1.5 \mathrm{~cm}$ x $15 \mathrm{~cm})$. The fraction $\quad R_{\mathrm{f}} \quad 0.52 \quad$ yielded 2-(piperidin-1-ylmethyl)-4-(3-(4(trifluoromethyl)phenyl)propyl)phenol (C) as a yellow oil (0.058 g, 86\%) (Figure 3).

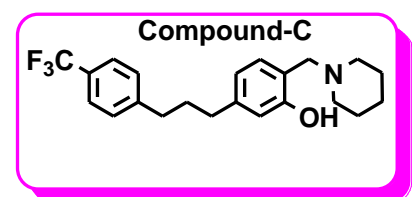

Figure 3. Structure of 2-(piperidin-1-ylmethyl)-4-(3-(4-(trifluoromethyl)phenyl)propyl)phenol.

${ }^{1} \mathrm{H}$ NMR (600 MHz, $\left.\mathrm{CDCl}_{3}, \mathrm{Me} 4 \mathrm{Si}\right) \delta$ ppm: 1.40 (2H, s, H-4'"), $1.61-1.51$ (4H, m, H3"', H-5"'), 1.90 - 1.75 (2H, m, H-2), 2.49 - 2.42 (2H, m, H-3), 2.63 - 2.56 (2H, m, H-1), 3.16 2.02 (4H, H-2"', H-6"'), 3.55 (2H, s, CH2), 6.66 (1H, d, J = 8.2 Hz, H-5"), 6.67 (1H, d, J = 2.2 Hz, H-2"), 6.88 (1H, dd, J = 8.2, 2.2 Hz, H-6"), 7.20 (2H, d, J = 8.0 Hz, H-2', H-6'), 7.45 (2H, $\left.\mathrm{d}, \mathrm{J}=8.0 \mathrm{~Hz}, \mathrm{H}-3{ }^{\prime}, \mathrm{H}-5{ }^{\prime}\right) ;{ }^{13} \mathrm{C} \mathrm{NMR}\left(150 \mathrm{MHz}, \mathrm{CDCl}_{3}, \mathrm{Me} 4 \mathrm{Si}\right) \delta \mathrm{ppm}: 24.0$ (C-4"') (Plate 45b and 45c), 25.9 (C-3"', C-5'"), 33.0 (C-2), 34.5 (C-1), 35.2 (C-3), 53.9 (C-2"', C-6"'), $62.2\left(\mathrm{CH}_{2}\right)$, 115.8 (C-2", C-5"), 121.4 (C-6"), $124.4\left(1 \mathrm{C}, \mathrm{q},{ }^{1} \mathrm{~J}_{\mathrm{C}-\mathrm{F}}=272.2 \mathrm{~Hz}, \mathrm{CF}_{3}\right), 125.2\left(2 \mathrm{C}, \mathrm{q},{ }^{3} \mathrm{~J}_{\mathrm{C}-\mathrm{F}}=3.9\right.$ $\left.\mathrm{Hz}, \mathrm{C}-3^{\prime}, \mathrm{C}-5^{\prime}\right), 128.1$ (1C, q, ${ }^{2} \mathrm{~J}_{\mathrm{C}-\mathrm{F}}=32.3 \mathrm{~Hz}, \mathrm{C}-4$ '), 128.3 (C-4"), 128.4 (C-6'), 128.7 (C-2'), 132.1 (C-1'), 146.6 (C-1"), 156.1 (C-3"); ${ }^{19} \mathrm{~F} \mathrm{NMR} \delta$ (282.4 MHz, $\left.\mathrm{CDCl}_{3}, \mathrm{C}_{6} \mathrm{~F}_{6}\right)-118.1$ (s, $\left.\mathrm{CF}_{3}\right)$; IR (neat): $v_{\max } \mathrm{cm}^{-1}=1067.03,1116.64,1323.65,1497.96,2936.14$; Found (TOF MS ES) $[\mathrm{M}+\mathrm{H}]^{+} 378.2043,\left(\mathrm{C}_{22} \mathrm{H}_{26} \mathrm{~F}_{3} \mathrm{NO}+\mathrm{H}^{+}\right): \mathrm{m} / \mathrm{z} 378.2045$; HPLC purity $87.3 \%, \mathrm{t}_{\mathrm{R}}=1.73 \mathrm{~min}$.

\section{Results and Discussion}

The collection of blood samples was done by tail bleeding at predetermined time intervals. The compounds having substituents $4-\mathrm{C}_{2} \mathrm{H}_{5}$ and $4-\mathrm{CF}_{3}$ were incubated invitro with human and mouse liver microsomes. In addition, the compounds were subjected to a parallel artificial membrane permeation assay. The results of in vitro studies revealed rapid 
absorption and hepatic metabolism of both the compounds $\mathrm{B}$ and $\mathrm{C}$. The maximum concentration of drug $\left(\mathrm{C}_{\max }\right)$ for the compounds $\mathrm{B}$ and $\mathrm{C}$ were observed to be $0.2 \pm 0.4 \mu \mathrm{M}$ and $0.7 \pm 0.3 \mu \mathrm{M}$, respectively and the elimination half-life of both compounds was $6.1 \mathrm{~h}$. Thus, we could successfully enhance the bioavailability from $3 \%$ to $25 \%$.

Pharmacokinetic studies were conducted for the compounds B \& C in C57/BL6 mice using reported method [7]. Doses of the compounds used are summarized in Table 1. The collection of blood samples was done by means of tail bleeding at pre-determined time intervals and the drug concentrations were determined with a LC/MS/MS method. During our study we found that the bioavailability of the compound was enhanced after the replacement of bulky groups at aromatic group. The in vitro studies were found to be more promising against Plasmodium falciparum. Fluorine at the $4^{\text {th }}$ position exhibited showed low bioavailability.

We synthesized different aminoalkylated diarylpropanes possessing 4- $\mathrm{CH}_{3}$ group and 4- $\mathrm{CF}_{3}$ group substitutions as an attempt to enhance the bioavailability. Pharmacokinetic studies were screened in C57BL/6 mice having with $15 \mathrm{mg} / \mathrm{kg} 4-\mathrm{CH}_{3}$ or $4-\mathrm{CF}_{3}$ administered orally and the $5 \mathrm{mg} / \mathrm{kg} 4-\mathrm{CH}_{3}$ or $4-\mathrm{CF}_{3}$ administered intravenously.

Table 1. Pharmacokinetic parameters for the synthesized compounds B and C following oral and i.v. administration in mice.

\begin{tabular}{|c|c|c|c|c|}
\hline \multicolumn{5}{|l|}{ Compound B } \\
\hline \multirow[t]{2}{*}{$\begin{array}{l}\text { Pharmacokinetic } \\
\text { parameter }\end{array}$} & \multicolumn{2}{|c|}{$\begin{array}{c}\text { IV administration } \\
(5 \mathrm{mg} / \mathrm{kg})\end{array}$} & \multicolumn{2}{|c|}{$\begin{array}{c}\text { Oral administration } \\
(15 \mathrm{mg} / \mathrm{kg})\end{array}$} \\
\hline & Average & SEM & Average & SEM \\
\hline $\mathrm{C}_{\max }(\mu \mathrm{M})$ & $\mathrm{n} / \mathrm{a}$ & $\mathrm{n} / \mathrm{a}$ & 0.180 & 0.010 \\
\hline $\mathrm{T}_{\max }(\min )$ & $\mathrm{n} / \mathrm{a}$ & $\mathrm{n} / \mathrm{a}$ & 0.500 & 0.00 \\
\hline Apparent half-life (h) & 6.033 & 0.338 & $\mathrm{n} / \mathrm{a}$ & $\mathrm{n} / \mathrm{a}$ \\
\hline $\begin{array}{l}\text { AUC } \\
(\min . \mu \mathrm{mol} / \mathrm{L})\end{array}$ & 142 & 17 & 31 & 9 \\
\hline $\mathrm{BA}(\%)$ & $\mathrm{n} / \mathrm{a}$ & $\mathrm{n} / \mathrm{a}$ & 7 & 2 \\
\hline \multicolumn{5}{|c|}{$\mathrm{n} / \mathrm{a}=$ not applicable } \\
\hline \multicolumn{5}{|l|}{ Compound C } \\
\hline \multirow[t]{2}{*}{$\begin{array}{l}\text { Pharmacokinetic } \\
\text { parameter }\end{array}$} & \multicolumn{2}{|c|}{$\begin{array}{c}\text { IV administration } \\
(5 \mathrm{mg} / \mathrm{kg})\end{array}$} & \multicolumn{2}{|c|}{$\begin{array}{c}\text { Oral administration } \\
(15 \mathrm{mg} / \mathrm{kg})\end{array}$} \\
\hline & Average & SEM & Average & SEM \\
\hline $\mathrm{C}_{\max }(\mu \mathrm{M})$ & $\mathrm{n} / \mathrm{a}$ & $\mathrm{n} / \mathrm{a}$ & 0.727 & 0.118 \\
\hline $\mathrm{T}_{\max }(\min )$ & $\mathrm{n} / \mathrm{a}$ & $\mathrm{n} / \mathrm{a}$ & 0.500 & 0.00 \\
\hline Apparent half-life (h) & 6.173 & 0.570 & $\mathrm{n} / \mathrm{a}$ & $\mathrm{n} / \mathrm{a}$ \\
\hline $\begin{array}{l}\mathrm{AUC} \\
(\mathrm{min} . \mu \mathrm{mol} / \mathrm{L})\end{array}$ & 239 & 32 & 178 & 7 \\
\hline $\mathrm{BA}(\%)$ & $\mathrm{n} / \mathrm{a}$ & $\mathrm{n} / \mathrm{a}$ & 25 & 1 \\
\hline
\end{tabular}

\section{Conclusions}

In summary, herein we report synthesis, characterization and anti-plasmodial activity of some aminoalkyl derivatives via the Mannich reaction. The in vitro absorption, metabolism and in vivo pharmacokinetics studies confirm the study revealed better absorption of both the compounds $\mathrm{B}$ and $\mathrm{C}$ as compared to $\mathrm{A}$. The in vitro and in vivo clearance values were found to correlate well. This has a triggered structure-activity relationship in an attempt to block metabolic sites without compromising the anti-malaria activity which will be a future guide of this series.

\section{Funding}

This research received no external funding. 


\section{Acknowledgments}

Authors are thankful to Technology Innovation Agency (TIA) for providing funding support under a research project - TA13 and also to CUT research office for the funding to the Master and Doctoral students at UDDR.

\section{Conflicts of Interest}

The authors declare no conflict of interest.

\section{References}

1. Tambo, E.; Adedeji, A.A.; Huang, F.; Chen, J.-H.; Zhou, S.-S.; Tang, L.-H. Scaling up impact of malaria control programmes: A tale of events in Sub-Saharan Africa and people's republic of China. Infectious Diseases of Poverty 2012, 1, 7-15, https://doi.org/10.1186/2049-9957-1-7.

2. Abdullahi, A.A.; Abubakar, A.D. Why it is difficult to eradicate malaria in Sub-Sahara Africa. Perspectives on Global Development and Technology 2019, 18, 269-285, https://doi.org/10.1163/15691497-12341516.

3. Aguzie, I.O.N. Pregnancy-associated malaria, challenges and prospects in Sub-Saharan Africa. Clinics Mother Child Health 2018, 15, 1-9, https://doi.org/10.4172/2090-7214.1000282.

4. Edelu, B.O.; Ndu, I.K.; Igbokwe, O.O.; Iloh, O.N. Severe falciparum malaria in children in Enugu, South East Nigeria. Nigerian Journal of Clinical Practice 2018, 21, 1349-1355.

5. Awoniyi, S.O.M.; Apata, T.G.; Igbalajobi, A.O.; Fakayode, S.B.; Sanusi, R.A.; Olorunfemi, B.J.; Ogunjimi, S.I. Malaria and agriculture: Evidence of relationship and labour/income loss among rural rice-farming households in Nigeria. Research on Crops 2018, 19, 353-364, https://doi.org/10.5958/23487542.2018.00052.9.

6. Mutabingwa, T.K. Artemisinin-based combination therapies (ACTs): best hope for malaria treatment but inaccessible to the needy! Acta Trop 2005, 95, 305-315, https://doi.org/10.1016/j.actatropica.2005.06.009.

7. Radke, J.B.; Burrows, J.N.; Goldberg, D.E.; Sibley, L.D. Evaluation of current and emerging antimalarial medicines for inhibition of Toxoplasma gondii growth in vitro. ACS Infectious Diseases 2018, 4, 1264-1274, https://doi.org/10.1021/acsinfecdis.8b00113.

8. van Huijsduijnen, R.H.; Wells, T.N. The antimalarial pipeline. Current Opinion in Pharmacology 2018, 42, 1-6, https://doi.org/10.1016/j.coph.2018.05.006.

9. Okombo, J.; Chibale, K. Recent updates in the discovery and development of novel antimalarial drug candidates. Medicinal Chemistry Communications 2018, 9, 437-453,https://doi.org/10.1039/C7MD00637C.

10. Achan, J.; Mwesigwa, J.; Edwin, C.P.; D'alessandro, U. Malaria medicines to address drug resistance and support malaria elimination efforts. Expert Review of Clinical Pharmacology 2018, 11, 61-70, https://doi.org/10.1080/17512433.2018.1387773.

11. Wilhelm, A.; Kendrekar, P.; Abay, E.; Bonnet, S.; Wiesner, L.; de Kock, C.; Swart, K.; van der Westhuizen, J. Syntheses and in vitro antiplasmodial activity of aminoalkylated chalcones and analogues. Frontiers in Natural Product Chemistry 2015, 78, 1848-1858, https://doi.org/10.1021/acs.jnatprod.5b00114.

12. Abay, E.T.; van der Westuizen, J.H.; Swart, K.J.; Gibhard, L.; Lawrence, N.; Dambuza, N.; Wilhelm, A.; Pravin, K.; Wiesner, L. Efficacy and pharmacokinetic evaluation of a novel anti-malarial compound (NP046) in a mouse model. Malarial Journal 2015, 14, 1-7, https://doi.org/10.1186/1475-2875-14-8.

13. Clarkson, C.; Campbell, W.E.; Smith, P. In vitro antiplasmodial activity of abietane and totarane diterpenes isolated from Harpagophytum procumbens (devil's claw). Planta Med 2003, 69, 720-724, https://doi.org/10.1055/s-2003-42778.

14. Clarkson, C.; Maharaj, V.J.; Crouch, N.R.; Grace, O.M.; Pillay, P.; Matsabisa, M.G.; Bhagwandin, N.; Smith, P.J.; Folb, P.I. In vitro antiplasmodial activity of medicinal plants native to or naturalised in South Africa. Journal of Ethnopharmacology 2004, 92, 177-191, https://doi.org/10.1016/j.jep.2004.02.011.

15. Liezl, G.; Kendrekar, P.; Efrem, A.; Anke, W.; Kenneth, S.; Nina, L.; Rosal, K.; Jan van der, W.; Peter, S.; Lubbe, W. Pharmacokinetics of aminoalkylated diarylpropanes NP085 and NP102. Antimicrobial Agents and Chemotherapy 2016, 60, 3065-3069, https://doi.org/10.1128/AAC.02104-15. 